\title{
Quantum Vacuum Contribution to the Momentum of the Dielectric Media.
}

\author{
A. Feigel \\ Electro-Optics Division, Soreq NRC, Yavne 81800, Israel
}

\begin{abstract}
Momentum transfer between matter and electromagnetic field is analyzed. The related equations of motion and conservation laws are derived using relativistic formalism. Their correspondence to various, at first sight self-contradicting, experimental data (the so called Abraham-Minkowski controversy) is demonstrated. A new, Casimir like, quantum phenomenon is predicted: contribution of vacuum fluctuations to the motion of dielectric liquids in crossed electric and magnetic fields. Velocities about $50 \mathrm{~nm} / \mathrm{s}$ can be expected due to the contribution of high frequency vacuum modes.

PACS numbers:
\end{abstract}


Electromagnetic radiation possesses energy, linear and angular momenta like any ordinary material object. As a consequence the light can exert mechanical forces on matter during the interaction. The forces associated with exchange of the linear and the angular momenta of light with material bodies were successfully measured already hundred years ago 1] 2] [3]. However, the fundamental question of the momentum associated with a photon in an optically dense medium is still under discussion 4] , despite that it was formulated in the beginning of the previous century. This problem arises from the discrepancy between Minkowski's [5] $G_{M}=\frac{1}{4 \pi c} \int d^{3} x D \times B$ and Abraham's [6] $G_{A}=\frac{1}{4 \pi c} \int d^{3} x E \times H$ results, where $E, H, D$ and $B$ are electric and magnetic fields and inductances correspondingly. Their difference is significant: while Minkowski's moment is directly proportional to the refractive index of the medium, the moment of Abraham possess inverse proportionality.

Minkowski momentum is considered by many as unacceptable, although it was shown by Jones, Richards and Leslie (JRL) that the recoil force of the light on a mirror immersed in liquid is directly proportional to the refractive index of the liquid [7]. This experiment was conducted twice with a 20 years period[8]. However most of the theoretical works are in favor of Abraham's expression (for a review, see refs. [9] [10]).

Abraham's momentum can be derived from the Poynting energy flow vector $S=\frac{c}{4 \pi} E \times H$ under assumption that all energy is purely electromagnetic and relates to the mass through the $U=m c^{2}$ relativistic formula. It corresponds to the relativistic requirement for direct proportionality of the energy and momentum flows (the symmetry of the electromagnetic stress tensor) 11]. Abraham's result is also supported by statistical physics approaches [12]. However, as far as we know, there are no experimental data that demonstrate the inverse dependence of the radiation pressure on the refractive index. This, at least, allows to conclude that the measured momentum is not purely electromagnetic.

The effective momentum of a photon in a dielectric medium consists of electromagnetic momentum and associated motion or even radiation of matter 9]. However separate identification of different parts proved itself to be non-trivial and sometimes led to contradictions with experimental data[8]. Blount [13] and Nelson[14] developed a Lagrangian formalism of the problem, using heuristic and microscopic averaging approaches correspondingly. They significantly clarified the picture by associating Abraham's expression with electromagnetic momentum and Minkowski's momentum with phonon like pseudomomentum. Still several questions remained open, especially a small discrepancy of Abraham's momentum with the 
expression derived in refs. [14] [15].

In this Letter the related Lagrangian and corresponding equations of motion are derived using relativistic formalism. In the case of liquid dielectric, interaction of the electromagnetic field with matter causes motion of the latter. Thus while Abraham's expression is indeed the momentum of the field, the measured momentum also includes the matter contribution and its value coincides with Minkowski's result. Afterwards the possible vacuum contributions to the motion of the matter are considered. Each electromagnetic mode possesses finite momentum, even in its ground state 16]. Thus modification of the modes by matter can alter the momentum of the vacuum. The latter generally vanishes due to counter propagating modes that cancel each other's contribution. This situation can be different however in materials that are temporally and spatially asymmetric.

The electromagnetic field in an optically dense medium is described by the Maxwell equations:

$$
\nabla \times H=\frac{1}{c} \frac{\partial D}{\partial t}, \nabla D=0, \nabla \times E=-\frac{1}{c} \frac{\partial B}{\partial t}, \nabla B=0 .
$$

The free electric $E$ and magnetic $B$ fields exist both inside and outside the matter. The matter response to the radiation is taken into account through derived fields $D$ and $H$, related to $E$ and $B$ by the dispersion relations. In the case of linear, non-dispersive dielectric medium they are given by:

$$
D=\varepsilon E, H=\frac{1}{\mu} B
$$

where $\varepsilon$ and $\mu$ are the dielectric and magnetic constants of the matter correspondingly.

The Lagrangian approach simplifies the investigation of light-matter interaction. The simplicity comes from the universality that allows to use the same approach to the system consisting of significantly different subsystems and the natural to the Lagrangian approach definition of the conservation laws. The Lagrangian which is equivalent to eqs. (11) and (21) is [17] [18]:

$$
L_{\text {Field }}=\int d^{3} x \frac{1}{4 \pi}\left(\frac{\varepsilon}{2} E^{2}-\frac{1}{2 \mu} B^{2}\right) .
$$

The first pair of the Maxwell equations (11) corresponds to the equations of motion:

$$
\frac{\partial}{\partial t} \frac{\partial L_{\text {Field }}}{\partial \frac{\partial A_{i}}{\partial t}}=\frac{\partial L_{\text {Field }}}{\partial A_{i}}, \frac{\partial}{\partial t} \frac{\partial L_{\text {Field }}}{\partial \frac{\partial \Phi}{\partial t}}=\frac{\partial L_{\text {Field }}}{\partial \Phi},
$$

while the second pair of (11) follows from definitions of the vector $A$ and the scalar $\Phi$ 
potentials:

$$
E=-\frac{1}{c} \frac{\partial A}{\partial t}-\nabla \Phi, B=\nabla \times A .
$$

The motion of the matter and especially its influence on the electromagnetic field must be taken into account in a combined matter-field Lagrangian $L_{M F}$. The linear dispersion relations (2) change in moving media to:

$$
D=\varepsilon E+\frac{\varepsilon \mu-1}{c \mu} v \times B, B=\mu H+\frac{\varepsilon \mu-1}{c} E \times v,
$$

where first order $v / c$ terms were taken into account 19] [20]. They follow from relativistic requirements and can be derived using the first order Lorentz transformations:

$$
\begin{aligned}
& E \rightarrow E+\frac{1}{c} v \times B, B \rightarrow B+\frac{1}{c} E \times v, \\
& D \rightarrow D+\frac{1}{c} v \times H, H \rightarrow H+\frac{1}{c} D \times v,
\end{aligned}
$$

relative to (2). These transformations can be applied directly to the Lagrangian (3). Substituting (17) into (31), keeping the first order $v / c$ terms and adding $\rho v^{2} / 2$ one obtains:

$$
L_{M F}=\int d^{3} x\left(\frac{1}{2} \rho v^{2}+\frac{1}{4 \pi}\left(\frac{\varepsilon}{2} E^{2}-\frac{1}{2 \mu} B^{2}+\frac{\varepsilon \mu-1}{\mu c} B(E \times v)\right)\right) .
$$

Since the liquid is assumed to be incompressible, it is described by its density $\rho$ and local velocity $v$ only. The equations of motion (44) of (9) are identical to the Maxwell equations with dispersion relations (6). The last term of (9) can be rewritten in an interaction $A j$ form, where the current $j$ is given by $\nabla \times(E \times v)(\varepsilon \mu-1) / \mu c$. The latter, at least for the non-magnetic $\mu=1$ case, can be obtained by microscopic averaging procedures [14] 11].

The Lagrangian (91) is explicitly independent of the space coordinates $x$, due to the homogeneity of space. Thus according to Noether theorem, the momentum[18]:

$$
G_{i}=\int d^{3} x\left(\frac{\partial L}{\partial v_{i}}-\frac{\partial L}{\partial \frac{\partial A_{j}}{\partial t}} \frac{\partial A_{j}}{\partial x_{i}}\right)
$$

is conserved. Substituting (19) into (10) one obtains:

$$
G=\int d^{3} x\left(\rho v+\frac{1}{4 \pi}\left(\frac{\varepsilon}{c} E \times B-\frac{\varepsilon \mu-1}{\mu c} E \times B\right)\right)=\int d^{3} x\left(\rho v+\frac{1}{4 \pi c} E \times H\right) .
$$

The corresponding angular momentum $l=x \times G$ becomes:

$$
l=\int d^{3} x\left(x \times \rho v+\frac{1}{4 \pi c} x \times E \times H\right) .
$$


Therefore the conserved linear (11) and angular (12) momenta consist of the matter and the Abraham's field terms. The correspondence between the conserved and the measured momenta follows from the analysis of the Lorentz force acting on material objects[11].

The $\rho v$ term can be obtained from the liquid's equation of motion:

$$
\frac{\partial}{\partial t} \frac{\partial L}{\partial v}=\frac{\partial L}{\partial R}
$$

where $R$ is the matter coordinate. Far from the boundaries, $\partial L / \partial R$ can be neglected, leading to:

$$
\rho v=\frac{\varepsilon \mu-1}{4 \pi \mu c} E \times B .
$$

This expression corresponds qualitatively to the pseudomomentum of ref. 14]. Substituting (14) into (11), one obtains $G=D \times B / 4 \pi c$, which was observed in the JRL experiments.

By analogy, the measured angular momentum is $l=x \times(D \times B)$. It can be separated into "spin" $D \times A$ and "orbital" $\sum D_{j}(x \times \nabla) A_{j}$ parts. The latter does not vanish only in the case of beams with specific wavefront distortions, e.g. Laguerre-Gaussian beams[21]. In contrast to the linear momentum, the spin part of the nearly plane wave is independent of dielectric properties of the medium. This was verified experimentally for microwave radiation 22].

The dielectric constant dependent angular momentum was observed inside a cylindrical capacitor filled with dielectric 23]]. A cylindrical capacitor was suspended on torsional levers inside some external magnetic field parallel to the axis of the cylinder. By applying voltage to the capacitor's walls a radial electric field was created. These independent fields possessed non-vanishing angular momentum, which was compensated by the motion of the capacitor itself. The observed 25] $x \times \rho v \propto(\varepsilon-1) l_{0}$, where $l_{0}$ corresponds to the $\varepsilon=1$ case, follows from (14) 24$]$.

Ashkin and Dziedzic observed that the liquid interface bends outwards the liquid in both cases when light enters and leaves the liquid[26]. Contrary to their measurement, the conservation law (11) predicts inward bending. Loudon recently arrived to the same conclusion by quantum analysis of the Lorentz force[4]. The results of ref. [26] were explained by the influence of ponderomotive forces [27], caused by strong focusing of the light in this experiment. These forces, also used for optical tweezers, are much stronger than contributions from the change of the radiation momentum on the boundary. 
Radiation forces can be caused even by redistribution of the energy between quantum vacuum and matter. Attraction of two parallel metal plates in vacuum was predicted by Casimir [28] and experimentally observed by Lamoreaux [29]. Electromagnetic field possesses finite energy even in the ground state, similar to the quantum harmonic oscillator. The presence of dielectric or metallic objects in space alternates the eigenstates of the electromagnetic field. The energy of such system depends on the specific arrangement of the objects and some rearrangement can be energetically favorable. Between two metal plates the low frequency electromagnetic modes are cut off by the boundary conditions. The smaller the separation the smaller is the effective energy of the system, consequently the plates attract each other. However, in Casimir case, no momentum is gained by the plate's center of the mass according to symmetry considerations. Moreover, to the best of our knowledge, the transfer of finite momentum from vacuum modes to matter was not considered yet.

The zero fluctuations contribution to the equation of motion (14) can be expected, since the moment of electromagnetic field, similar to its energy, is a quadratic function of $E$ and $B$. Vacuum contribution can not occur, neither in time even media nor in spatially symmetrical time-odd (Faraday) materials, due to the self compensation of counterpropagating modes. Therefore both time and spatial asymmetries are required.

The break of both spatial and time symmetries occur naturally in magnetoelectric materials [30], 31]. The dispersion relations for magnetoelectrics are:

$$
D=\widehat{\varepsilon} E+\widehat{\chi} H, B=\widehat{\mu} H+\widehat{\chi}^{T} E .
$$

The same dispersion can be created artificially by applying external electric and magnetic fields [32]. In this case, the dielectric properties of the medium $\widehat{\varepsilon}, \widehat{\mu}$ and $\widehat{\chi}$ depend on the external fields $E_{\text {ext }}$ and $B_{\text {ext }}$. For the specific case of perpendicular electric and magnetic fields acting on isotropic material [33] [34]:

$$
\widehat{\varepsilon}=\left(\begin{array}{ccc}
\varepsilon & 0 & 0 \\
0 & \varepsilon & 0 \\
0 & 0 & \varepsilon
\end{array}\right) \widehat{\mu}=\left(\begin{array}{ccc}
\mu & 0 & 0 \\
0 & \mu & 0 \\
0 & 0 & \mu
\end{array}\right) \widehat{\chi}=\left(\begin{array}{ccc}
0 & \chi_{x y} & 0 \\
\chi_{y x} & 0 & 0 \\
0 & 0 & 0
\end{array}\right)
$$

For light propagating along $z=E_{\text {ext }} \times B_{\text {ext }}$ direction, substituting (15) and (16) into Maxwell equations (11) one obtains [34]:

$$
n_{\vec{k}, 1}=\sqrt{\varepsilon \mu}+\chi_{x y}, n_{\vec{k}, 2}=\sqrt{\varepsilon \mu}-\chi_{y x}, n_{-\vec{k}, 1}=-\sqrt{\varepsilon \mu}+\chi_{x y}, n_{-\vec{k}, 2}=-\sqrt{\varepsilon \mu}-\chi_{y x} .
$$


and corresponding modes $\left(E_{x}, E_{y}, B_{x}, B_{y}\right)$ :

$$
(1,0,0, \sqrt{\varepsilon \mu}),(0,1,-\sqrt{\varepsilon \mu}, 0),(1,0,0,-\sqrt{\varepsilon \mu}),(0,1, \sqrt{\varepsilon \mu}, 0) .
$$

In the case of magnetoelectrics (15), the term $\frac{1}{\mu} B \widehat{\chi}^{T} E$ must be added to the Lagrangian (91). Using (71) one obtains:

$$
L_{M E}=L_{F M}+\int \frac{d^{3} x}{4 \pi}\left(\frac{1}{\mu} B \widehat{\chi}^{T} E+\frac{1}{\mu c} B \widehat{\chi}^{T}(v \times B)+\frac{1}{\mu c}(E \times v) \widehat{\chi}^{T} E\right) .
$$

Equations of motion (41) correspond to the dispersion relations (15) in moving media, while (13) becomes:

$$
\rho^{0} v=\frac{1}{4 \pi}\left(\frac{\varepsilon \mu-1}{\mu c} E \times B+\frac{1}{\mu c} E \times\left(\widehat{\chi}^{T} E\right)-\frac{1}{\mu c} B \times(\widehat{\chi} B)\right) .
$$

The non-compensating moment of a pair of counterpropagating modes in $z$ direction is $\Delta p=\left(\chi_{x y}-\chi_{y x}\right)(1+\varepsilon \mu) /(2 \pi \mu c)$. It is obtained by substitution of (18) into (20). Taking into account all contributing modes and $\Delta p(\theta)=\Delta p \cos \theta$, we obtain:

$$
v=\frac{1}{\rho} \frac{1}{2 \pi} \frac{1+\varepsilon \mu}{\mu c} \int_{0, \infty}^{\pi / 2,2 \pi / \omega_{\text {cut }}} \Delta n \cos \theta k^{2} E^{2} \frac{d k}{\pi^{2}} \sin \theta d \theta,
$$

where $\Delta n=\left(\chi_{x y}-\chi_{y x}\right)$. The vacuum $E_{v a c}^{2}=\hbar \omega / 2$, thus (21) becomes:

$$
v=\frac{1}{32 \pi^{3}} \frac{1}{\rho} \Delta n \frac{1+\varepsilon \mu}{\mu} \frac{\hbar \omega_{c u t}^{4}}{c^{4}} .
$$

This expression is significantly different from the Casimir effect, since it is powered by the high frequency cut-off. The latter makes it more similar to the Lamb shift phenomenon.

This effect (22) can be evaluated quantitatively by the estimation of the value of $\Delta n$ from the known experimental data. In crossed external magnetic $B_{\text {ext }}$ and electric $E_{\text {ext }}$ fields, $\Delta n$ is proportional to magnetoelectric susceptibility $\beta_{\perp}[35]$ :

$$
\Delta n \approx\left(\frac{3}{2} \beta_{\perp}-\frac{1}{2} \beta_{\|}\right) E_{\text {ext }} B_{\text {ext }} l_{0}^{-1} \approx \beta_{\perp} E_{\text {ext }} B_{\text {ext }} l_{0}^{-1}
$$

where $l_{0} \approx 0.3 \mathrm{~nm}$ is the characteristic interatomic distance. This result follows from the spherically symmetric system's fourth order energy terms $L=1 / 4 \beta_{\perp} E^{2} B^{2}+$ $1 / 4\left(\beta_{\|}-\beta_{\perp}\right)(E B)^{2}$ and $D=\partial L / \partial E$ relation. The $\chi_{x y}+\chi_{y x} \approx 1 e-11$ was recently observed [32] by measurement of magnetoelectric linear birefringence (17) in external electric $E_{\text {ext }}=1 e 5 \mathrm{~V} / \mathrm{m}$ and magnetic $B_{\text {ext }}=17 \mathrm{~T}$ fields. The contribution of the non-local 
terms 36] 32] to $\Delta n$, leading to $\Delta n \propto 1 / \lambda$, can significantly increase the value of (22). However, taking into account that $\Delta n \approx \chi_{x y}-\chi_{y x} \approx \chi_{x y}+\chi_{y x}$, the $\beta_{\perp} \approx 0.1 a . u$., which corresponds to the experimentally observed $\Delta n \approx 1 e-11[32]$ according to (23) , is in the range of theoretical predictions [37] 38]. Therefore $\Delta n$ is assumed to be constant in the (21) integral.

According to (22) $v_{v a c} \approx 50 \mathrm{~nm} / \mathrm{s}$ in external fields $E_{\text {ext }}=1 e 5 \mathrm{~V} / \mathrm{m}$ and $B_{\text {ext }}=17 T$. The cut-off frequency $\omega_{\text {cut }}$ was chosen to correspond to a wavelength $\lambda=2 \pi c / \omega \approx 0.1 \mathrm{~nm}$, since for higher frequencies the molecular polarization vanishes. Density $\rho \approx 1 \mathrm{e} 3 \mathrm{~kg} / \mathrm{m}^{3}$, $\Delta n \approx 1 e-11$ and dielectric constant $\varepsilon \approx 1.5$ were assumed. The contribution of the static field (14) is $v_{\text {stat }} \approx 20 \mathrm{~nm} / \mathrm{s}$. In JRL experiment the estimated velocity from (14) was $v_{\text {laser }} \approx 1 e-15 \mathrm{~m} / \mathrm{s}$ (the laser beam intensity was about $1 e 5 \mathrm{~W} / \mathrm{m}^{2}$ ). Velocity $v_{\text {vac }}$ is not only greater than $v_{\text {stat }}$ and $v_{\text {laser }}$, but may also possess the opposite sign. The latter follows from substitution of ref. [37] results into (23). The experimental measurement of (22) requires effective homogeneity of the matter. Otherwise eqs. (14) and (20) are not valid. Thus the region of the crossed fields must be produced locally, similar to the laser beam in JRL experiment. It can be done for instance by immersing a capacitor's electrodes inside the liquid.

Any liquid possess viscosity, causing decay in time of any artificially created flow. The dissipation processes can be taken into account empirically by adding correspondent viscous forces $\eta \Delta v$ to the equation of motion (13):

$$
\rho \frac{\partial v}{\partial t}=\eta \Delta v
$$

while (14) defines the boundary conditions. According to (14), the Gaussian laser beam in JRL experiment generated Gaussian flow. Eq. (24), in such case, becomes an ordinary diffusion equation. The initially gained momentum of the liquid "diffuses" in a plane perpendicular to the propagation direction. Thus the characteristic decay time is $t_{d} \approx l_{\text {mirror }}^{2} \rho / \eta \approx 10 s$, where the mirror's size $l_{\text {mirror }} \approx 0.25 \mathrm{~cm}$ and the density to viscosity ratio $\rho / \eta \approx 200 \mathrm{~s} / \mathrm{cm}^{2}[8]$. Radiation pressure in liquids was studied on the time scales comparable with $t_{d}[8]$. Longer timescale observations can separate the electromagnetic and liquid flow contributions to the measured force, because the measured momentum (11), in the case of static liquid, corresponds to Abraham's momentum. It is also feasible in an experiments with strong overlap between incident and reflected beams, e.g. measurement in a tube. The motion of matter 
can not occur under symmetric illumination, thus the measured force must be independent of the liquid's refractive index.

In conclusion relativistic formalism was applied for light-matter Lagrangian derivation. Equations of motion were obtained and their correspondence to the Abraham-Minkowski controversy related experimental data was demonstrated. The received results correspond to Abraham's predictions, while Minkowski's momentum can be obtained from (9) without its last "motion of the matter" term. Therefore the origin of the controversy lies in the underestimation of the fact that the field-matter interaction is impossible without the motion of the latter. The vacuum fluctuations induced flow in dielectric liquids with $v_{v a c} \approx 50 \mathrm{~nm} / \mathrm{s}$ was predicted in external crossed electric and magnetic fields. The significant property of this phenomenon is the high frequency vacuum modes contribution, similar to the Lamb shift effect. It can be used in future as an investigating tool for zero fluctuations. Other possible applications lie in fields of microfluidics or precise positioning of microobjects, e.g. cold atoms or molecules. Initial experimental verifications can be based on artificially created random fields.

The helpful discussions with A. Englander, B. Sfez and Y. Siberberg are gratefully acknowledged.

[1] P. Lebedew, Ann. Phys. 6, 433 (1901).

[2] E. F. Nichols and G. F. Hull, Phys. Rev. 13, 307 (1901).

[3] R. A. Beth, Phys. Rev. 50, 115 (1936).

[4] R. Loudon, J. Mod. Optics 49, 821 (2002).

[5] H. Minkowski, Nachr. Ges. Wiss. Göttingen 53 (1908); Math. Ann. 68, 472 (1910).

[6] M. Abraham, Rend. Pal. 28, 1 (1909): 30, 33 (1910).

[7] R. V. Jones and J. C. S. Richards, Proc. Roy. Soc. A 221, 480 (1954).

[8] R. V. Jones and B. Leslie, Proc. R. Soc. Lond. A 360, 347 (1978).

[9] R. Peierls, More Surprises in Theoretical Physics (Princeton Univ. Press, Princeton, 1991).

[10] I. Brevik, Physics Reports 52, 133 (1979).

[11] J. D. Jackson, Classical Electrodynamics (Wiley, New- York, 1998) ps.256-262.

[12] S. R. de Groot and L. G. Suttorp, Foundations of Electrodynamics (North-Holland, Amster- 
dam, 1972).

[13] E. I. Blount, Bell Laboratories Memorandum, 71-1111-33 (1971).

[14] D. F. Nelson, Phys. Rev. A 44, 3985 (1991).

[15] R. Loudon, L. Allen, and D. F. Nelson, Phys. Rev. E 55, 1071 (1997).

[16] L. Mandel and E. Wolf, Optical Coherence and Quantum Optics (Cambridge University Press, 1995) p.485.

[17] L. D. Landau and E. M. Lifshitz, The classical theory of fields, 4th ed. (Pergamon, Oxford, 1975).

[18] H. Goldstein, Classical Mechanics (Addison-Wesley, Reading, 1980).

[19] L. D. Landau, E. M. Lifshitz, and L. P. Pitaevskii, Electrodynamics of Continuous Media (Pergamon, New York, 1984).

[20] W. Pauli, Theory of Relativity (Pergamon, New York, 1967).

[21] M. Padgett and L. Allen, Cont. Phys. 41, 275 (2000).

[22] M. Kristensen and J. P. Woerdman, Phys. Rev. Lett. 72, 2171 (1994).

[23] G. M. Graham and D. G. Lahoz, Nature 285, 154 (1980).

[24] In the case of small particles or even molecules the conservation law (11) coincides with (14), where $E(\varepsilon \mu-1) / 4 \pi \mu c$ is replaced by the correspondent polarization. It can be easily shown for the small spherical or cylindrical particles in the external fields. Although the assumption about matter homogeneity is not valid and (13) can not be used, intuitively it can be understood considering the Lorentz force acting on a small object in alternating fields.

[25] G. B. Walker, D. G. Lahoz, and G. Walker, Can. J. Phys. 53, 2577 (1975).

[26] A. Ashkin and J. M. Dziedzic, Phys. Rev. Lett. 30, 139 (1973).

[27] J. P. Gordon, Phys. Rev. A 8, 14 (1973).

[28] H. B. G. Casimir, Proc. K. Ned. Akad. Wet. 51, 793 (1948).

[29] S. K. Lamoreaux, Phys. Rev. Lett. 78, 5 (1997).

[30] R. Fuchs, Philos. Mag. 11, 647 (1965).

[31] T. H. O’Dell, Philos. Mag. 11, 921 (1965).

[32] T. Roth and G. L. J. A. Rikken, Phys. Rev. Lett. 88, 063001 (2002).

[33] G. L. J. A. Rikken and C. Rizzo, Phys. Rev. A 63, 012107 (2000).

[34] A. Figotin and I. Vitebsky, Phys. Rev. B 63, 066609 (2001).

[35] A. D. Buckingham and J. A. Pople, Proc. Cambridge Phil. Soc. 53, 262 (1957). 
[36] H. J. Ross, B. S. Sherborne, and G. E. Stedman, J. Phys. B 22, 459 (1989).

[37] J. D. Lyons and R. P. Hurst, Phys. Rev. 162, 38 (1967).

[38] E. B. Graham and R. E. Raab, Mol. Phys. 52, 1241 (1984). 